\title{
ERP/EAI System Issues and Answers: A Research Journey
}

\author{
Gail Corbitt, Marinos Themistocleous2 and Zahir Irani2 \\ California State University, Chico, USA \\ ${ }_{2}$ Brunel University, London UK
}

Information Technology (IT) is being increasingly used to automate business processes at strategic, tactical and operational levels. However, traditionally these applications have not developed or been implemented in a coordinated way, but increasingly evolved as a result of technological innovation and business needs. As a result, the IT infrastructure in many organizations consists of autonomous and in many cases heterogeneous solutions that have often been unable to evolve with the business needs of the organization. The effect of this has caused various integration problems as applications can not co-operate, and disparate IT solutions can not be integrated together.

To address of these concerns, the early 1990's saw the emergence of Enterprise Resource Planning (ERP) systems. ERPs were introduced as integrated suites that automate core corporate activities such as manufacturing, finance, human resources and logistics. Such systems allow a company to share common data and practices across the enterprise, and support the access of information in real-time environments, which is proving paramount to the success of certain business sectors, such as the process industry and supply chain management - logistics and distribution in particular. According to Davenport (1998) ERP solutions are designed to solve the fragmentation of information in businesses, and integrate all the information flowing within a company.

Nonetheless, after more than a decade of ERP, the need for integration still exists as ERP packages can not effectively address the problem of autonomous applications that need to co-exist alongside ERP. The research area, is being expanded to include, not only the impact of ERP from a technological, human and organizational perspective, but also the integration of ERP with other systems through integrated EAI (Enterprise Application Integration).

There remains a constant thread with regards to the use of ERP, with much concern centered on how such systems operate alongside each other as well as with other disparate systems. Much of this initial concern has been addressed through a new EAI paradigm in software development, (Irani et al., 2003; Themistocleous et al., 2004). Despite the apparent importance of EAI for organizations, the topic remains somewhat under-researched and represented in the academic normative literature. This scant literature is attributed to EAI technologies being in their infancy, as well as the interchangeability in terminology; Application Integration, Web Services, Enterprise Application Integration.

This year 13 papers were submitted for this mini-track, which has now been running in one form or another for over 5 years. Our thanks go to the many reviewers who have helped us [the mini-track chairs] develop the final program and structure to the minitrack. The papers were of a high standard and we believe that this year's specific mini-track on 'ERP /EAI Systems, Issues and Answers' will continue to be highly beneficial to the ERP and information systems community. It will enable new and different insights into ERP and EAI to be viewed in a more holistic and integrated manner. This year's mini-track deals with a plethora of exciting issues that explore both positive and interpretive paradigms, so there is something for everyone. The five papers that were accepted for presentation continue to weave a coherent story to expose some of the underlying issues within the area of ERP and EAI. We look forward to a lively discussion at the conference in January 2006.

Davenport, T. 1998. 'Putting the Enterprise into the Enterprise System', Harvard BusinessReview, July-August 1998): 121-131.

Irani Z, Themistocleous $\mathrm{M}$ and Love P.E.D. 2003. 'The impact of enterprise application integration on information system lifecycles'. Information and Management, 41(2): 177-188.

Themistocleous M, Irani Z, Love P.E.D. 2004. 'Evaluating the Integration of Supply Chain Information Systems'. European Journal of Operational Research, 159(2): 393-40 